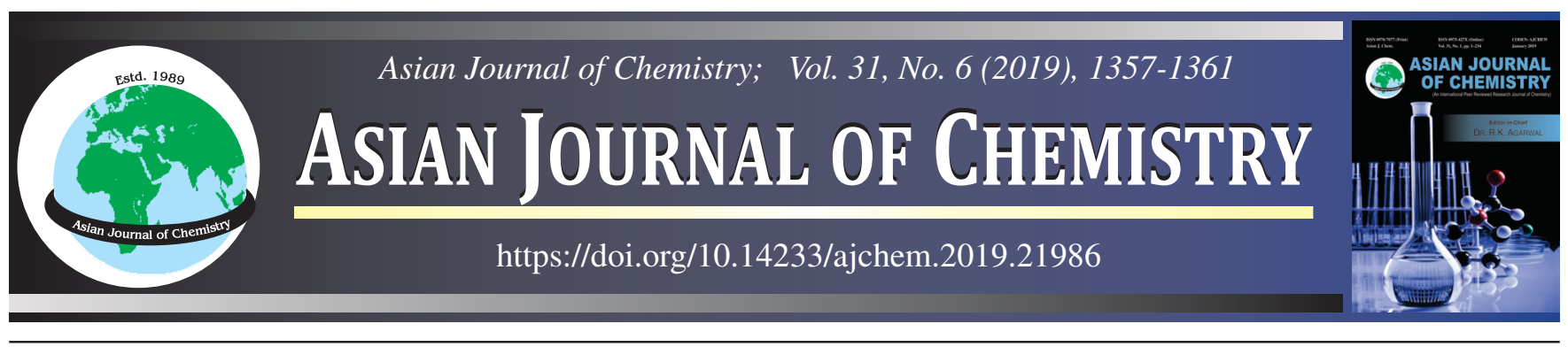

\title{
A Facile Solvent-Free Route for One-Pot Multicomponent Synthesis of Benzylpyrazolyl Coumarins Derivatives in Presence of Effective Synergetic Catalytic System
}

\begin{abstract}
NAREndRa R. KAMBLE ${ }^{1, *}$ and VinOD T. KAMBLE ${ }^{2}$
${ }^{1}$ Department of Chemistry, PDEA's Waghire College, Saswad-412301, India

${ }^{2}$ Organic Chemistry Research Laboratory, Department of Chemistry, Institute of Sciences, Nagpur-440008, India

*Corresponding author: E-mail: narendra1499@gmail.com

Received: 7 February 2019;

Accepted: 11 March 2019;

Published online: 29 April 2019;

AJC-19380

A combinatorial library of benzylpyrazolyl coumarin derivatives have been synthesized by a green one-pot four-component reaction
between aryl hydrazine/hydrazine hydrate $(\mathbf{1})$, ethyl acetoacetate $(\mathbf{2})$, aromatic aldehydes $(\mathbf{3})$ and 4-hydroxycoumarin $(\mathbf{4})$ catalyzed by
niobium pentachloride with silver salt under solvent-free conditions has been developed. Experimental simplicity, simple work-up procedure
and solvent-free reaction condition at room temperature are important features of the present protocol.
Keywords: Niobium pentachloride, Silver salt, Benzylpyrazolyl coumarin.
\end{abstract}

\section{INTRODUCTION}

Recently, green chemistry has received more attention due to its great applications and approaches that aim to prevent pollution and reduce the use of the waste materials that affect the human health and environment. Therefore, a number of chemists focused on designing of methodologies to create chemical protects without toxicity to human health. In this sense, a green chemistry focuses mainly on chemical syntheses using novel synthetic methods that provide affordable protects and services to the human and society [1-5]. To enhance chemical processes and environment friendly protects, the chemists working in green chemistry carry out multicomponent reactions that offer great advantages in the green chemistry, in which various flow one-pot transformations occur in the same step to offer the product without a need to segregate or isolate any compounds produced in the intermediate steps [6-8].

The important synthesized compounds by multicomponent reactions were these derivatives containing coumarins and pyrazolone nucleus. 3-Substituted 4-hydroxycoumarin, especially 3-benzyl substituted 4-hydroxycoumarin derivatives that are of extreme significance because they exist abundantly in natural products and exhibit a wide range of biological activities as they are used in medicinal scaffolds, namely warfarin, phenprocoumon, coumatetralyl, carbochromen, bromadialone, offering antibacterial, anti-HIV [9], antiviral [10], anticoagulant [11], antioxidant [12] and anticancer activities [13]. Moreover, pyrazolones are regarded as important structural nuclei in many drugs such as convenient antipyretic and analgesic drugs [14], whereas edaravone (MCI-186) has been useful for curing brain $[15,16]$ and myocardial ischemia [17]. In addition, pyrazolones have kinase inhibitory effects, particularly in the case of enzymes which catalyze both phosphorylation of serine and threonine in proteins. They are also used for treating diseases related to these enzymes, such as rheumatoid arthritis, psoriasis, bone loss, cancer and other proliferative diseases including antifungal [18], antimycobacterial [19,20], antibacterial [21], anti-inflammatory [22], antitumor [23], gastric secretion stimulatory [24], antidepressant [25], antifilarial activities [26] and antitubercular activities [27].

In the present work, a novel route was used for the synthesis of benzylpyrazolyl coumarins (5) with high yields by one pot four component condensation reaction of hydrazine (1), ethyl acetoacetate (2), aromatic aldehyde (3) and 4-hydroxycoumarin (4) reacted in the presence of a mixture of $\mathrm{NbCl}_{5}$ and $\mathrm{AgClO}_{4}$ as Lewis acid and a combined catalyst under room temperature and stirring conditions, in short reaction time and environmentally benign.

This is an open access journal, and articles are distributed under the terms of the Creative Commons Attribution-NonCommercial-ShareAlike 4.0 (CC BY-NC-SA 4.0) International License which allows readers to freely read, download, copy, distribute, print, search, or link to the full texts of its articles and to use them for any other lawful non-commercial purpose as long as the original source is duly acknowledged. 


\section{EXPERIMENTAL}

General procedures for the synthesis of benzylpyrazolyl coumarin derivatives: In a mixture of $5 \mathrm{~mol}$ of ethanol and $5 \mathrm{~mL}$ of water, phenyl hydrazine $(1 \mathrm{mmol})$, ethyl acetoacetate (1 mmol), aromatic aldehyde (1 mmol) and 4-hydroxycoumarin ( $1 \mathrm{mmol}$ ), $1 \mathrm{~mol} \mathrm{\%} \mathrm{of} \mathrm{NbCl}_{5}$ and $3 \mathrm{~mol} \%$ of $\mathrm{AgClO}_{4}$ were dissolved and stirred at room temperature. After completing the reaction monitor by thin-layer chromatography, the reaction mixture was left to be cooled at room temperature. Then, the reaction mixture was poured into cold water and the crystalline product was filtered. After that, the product was washed by water for 2-3 times and, then, dried at the room temperature and recrystallized from hot ethanol to afford the corresponding benzylpyrazolyl coumarin derivatives. To recover the catalyst from the filtrate, the aqueous layer was evaporated under reduced pressure to yield the final product. All the products were characterized by IR, ${ }^{1} \mathrm{H}$ NMR and mass spectra.

1,2-Dihydro-4-((4-hydroxy-2-oxo-2H-chromen-3-yl) (phenyl) methyl)-5-methyl-2phenyl-1H-pyrazol-3(2H)-3one (5a): Yield: $80 \%$, white crystalline solid, m.p.: 230-232 ${ }^{\circ} \mathrm{C}$, IR $\left(\mathrm{KBr}, v_{\max }, \mathrm{cm}^{-1}\right)$ : 3062, 2803, 1764, 1720, 1668, 1450, 1174, 1028,750. ${ }^{1} \mathrm{H}$ NMR (400 MHz, DMSO) $\delta$ ppm: 1.75 (s, $1 \mathrm{H}), 2.21(\mathrm{~s}, 3 \mathrm{H}), 5.74(\mathrm{~s}, 1 \mathrm{H}), 7.73-7.80(\mathrm{~m}, 4 \mathrm{H}), 7.18-7.35(\mathrm{~m}$, $5 \mathrm{H}), 5.74-7.69(\mathrm{~m}, 5 \mathrm{H})$. LC-MS (ESI) $\mathrm{m} / z\left[\mathrm{M}^{+}+\mathrm{H}\right]=388.1$. $\mathrm{C}_{26} \mathrm{H}_{20} \mathrm{~N}_{2} \mathrm{O} 4([\mathrm{M}+\mathrm{H}])$ 424.1, found: 424.1. Elemental analysis calcd. (found) \%: C 73.17 (73.15); H 4.25 (4.42); N 6.00 (6.22).

1,2-Dihydro-4-((4-hydroxy-2-oxo-2H-chromen-3-yl)(4-nitrophenyl)methyl)-5-methyl-2-phenylpyrazol-3-one (5b): Yield: $92 \%$, pale yellow crystalline solid, m.p.: 248$250{ }^{\circ} \mathrm{C}$, IR (KBr, $\left.v_{\max }, \mathrm{cm}^{-1}\right): 3073,1649,1608,1560,1525$, 1178, 1039, 748. ${ }^{1} \mathrm{H}$ NMR (400 MHz, DMSO) $\delta$ ppm: 2.65 (s, $3 \mathrm{H}), 6.12$ (s, 1H), 7.46-7.78 (m, 9H), 7.94-8.97 (m, 5H). LC-MS (ESI) $m / z\left[\mathrm{M}^{+}+\mathrm{H}\right]=469 . \mathrm{C}_{26} \mathrm{H}_{19} \mathrm{~N}_{3} \mathrm{O}_{6}([\mathrm{M}+\mathrm{H}])$ 469.6, found: 469.1. Elemental analysis calcd. (found) \%: C 66.02 (66.55); H 4.28 (4.11); N 8.25 (8.94).

1.2-Dihydro-4-((4-hydroxy-2-oxo-2H-chromen-3-yl)(3-nitrophenyl)methyl)-5methyl-2-phenyl-1H-pyrazol-3(2H)-3-one (5c): Yield: 90 \%, yellow crystalline solid, m.p.: 200-202 ${ }^{\circ} \mathrm{C}$, IR (KBr, $\left.v_{\max }, \mathrm{cm}^{-1}\right)$ : 2918, 1764, 1681, 1521, 1458, 1173, 1043, 756. ${ }^{1} \mathrm{H}$ NMR (400 MHz, DMSO) $\delta$ ppm: 2.20 (s, $3 \mathrm{H}), 5.79(\mathrm{~s}, 1 \mathrm{H}), 7.05-7.07(\mathrm{~m}, 4 \mathrm{H}), 7.11-7.20(\mathrm{~m}, 2 \mathrm{H}), 7.24-$ 7.31 (m, 2H), 7.41-7.49 (m, 2H), 7.82-7.89 (m, 2H). LC-MS (ESI) $m / z\left[\mathrm{M}^{+}+\mathrm{H}\right]=434.1 . \mathrm{C}_{26} \mathrm{H}_{19} \mathrm{~N}_{3} \mathrm{O}_{6}([\mathrm{M}+\mathrm{H}]) 469.1$, found: 469.8. Elemental analysis calcd. (found) \%: C 66.12 (66.33); H 4.20 (4.00); N 8.95 (8.96).

1,2-Dihydro-4-((4-hydroxy-2-oxo-2H-chromen-3-yl)(p-tolyl)methyl)-5-methyl-2 phenylpyrazol-3-one (5d): Yield: $92 \%$, white crystalline solid, m.p. 222-224 ${ }^{\circ} \mathrm{C}$, IR (KBr, $\left.v_{\max }, \mathrm{cm}^{-1}\right): 3081,1649,1610,1494,1184,1031,752 .{ }^{1} \mathrm{H}$ NMR (400 MHz, DMSO) $\delta$ ppm: 2.18 (s, 3H), 2.34 (s, 3H), 5.68 (s, $1 \mathrm{H}), 6.92-7.19(\mathrm{~m}, 7 \mathrm{H}), 7.31-7.41(\mathrm{~m}, 4 \mathrm{H}), 7.58-7.61$ (d, $J=$ $7.8 \mathrm{~Hz}, 2 \mathrm{H}), 7.75-7.78(\mathrm{~m}, 1 \mathrm{H})$. LC-MS (ESI) $m / z .\left[\mathrm{M}^{+}+\mathrm{H}\right]=$ 438.1. $\mathrm{C}_{27} \mathrm{H}_{22} \mathrm{~N}_{2} \mathrm{O}_{4}([\mathrm{M}+\mathrm{H}])$ 438.5, found: 438.1. Elemental analysis calcd. (found) \%: C 73.06 (73.99); H 5.86 (5.07); N 6.39 (6.42).

4-((4-Chlorophenyl)(4-hydroxy-2-oxo-2H-chromen-3yl)(phenyl)methyl)-5-methyl-2phenyl-1H-pyrazol-3(2H)-3- one (5e): Yield: $93 \%$, white crystalline solid, m.p.: 225-227 ${ }^{\circ} \mathrm{C}, \mathrm{IR}\left(\mathrm{KBr}, v_{\max }, \mathrm{cm}^{-1}\right)$ : 3064, 1651, 1602, 1492, 1177, 1095, 752. ${ }^{1} \mathrm{H}$ NMR (400 MHz, DMSO) $\delta$ ppm: 2.44 (s, 3H), 5.72 (s, 1H), 7.17-7.24 (m, 8H), 7.34-7.50 (m, 3H), 7.58-7.91 (m, 3H). LC-MS (ESI) $m / z\left[\mathrm{M}^{+}+\mathrm{H}\right]=461.1 . \mathrm{C}_{26} \mathrm{H}_{19} \mathrm{~N}_{2} \mathrm{O}_{4} \mathrm{Cl}([\mathrm{M}+\mathrm{H}])$ 458.1, found: 458.8. Elemental analysis calcd. (found) \%: C 68.55 (68.07), H 4.57 (4.20); N 6.20 (6.13).

4-((4-Fluorophenyl)(4-hydroxy-2-oxo-2H-chromen-3yl)methyl)-1,2dihydro-5-methyl-2-phenylpyrazol-3-one (5f): Yield: $93 \%$, white crystalline solid, m.p.: $240-242{ }^{\circ} \mathrm{C}$, IR (KBr, $\left.v_{\max }, \mathrm{cm}^{-1}\right): 3081,1656,1607,1566,1499,1187,1040$, 754. ${ }^{1} \mathrm{H}$ NMR (400 MHz, DMSO) $\delta$ ppm: 2.36 (s, 3H), 5.70 (s, 1H), 6.80-6.86 (m, 2H), 7.09-7.19 (m, 5H), 7.31-7.42 (m, 3H), 7.57-7.60 (d, $J=8.1 \mathrm{~Hz}, 2 \mathrm{H}), 7.76-7.79(\mathrm{~m}, 1 \mathrm{H}), 12.03(\mathrm{bs}, 1 \mathrm{H})$. LC-MS (ESI) $m / z\left[\mathrm{M}^{+}+\mathrm{H}\right]=442.1 . \mathrm{C}_{26} \mathrm{H}_{19} \mathrm{~N}_{2} \mathrm{O}_{4} \mathrm{~F}([\mathrm{M}+\mathrm{H}])$ 442.6, found: 442.1. Elemental analysis calcd. (found) \%: C 70.58 (70.61), H 4.33 (4.35) and N 6.33 (6.36).

1,2-Dihydro-4-((4-hydroxy-2-oxo-2H-chromen-3-yl)(4-hydroxyphenyl)methyl)-5-methyl-2-phenyl-1 $H$-pyrazol3(2H)-3-one (5g): Yield: $88 \%$, white cream solid, m.p.: 220$222{ }^{\circ} \mathrm{C}$, IR (KBr, $\left.v_{\max }, \mathrm{cm}^{-1}\right): 3501,1656,1607,1566,1499$, 1187, 1040, 754. ${ }^{1} \mathrm{H}$ NMR (400 MHz, DMSO) $\delta \mathrm{ppm}: 2.36(\mathrm{~s}$, $3 \mathrm{H}), 5.70$ (s, 1H), 6.80-6.86 (m, 2H), 7.09-7.19 (m, 5H), 7.31$7.42(\mathrm{~m}, 3 \mathrm{H}), 7.57-7.60(\mathrm{~d}, J=8.1 \mathrm{~Hz}, 2 \mathrm{H}), 7.76-7.79(\mathrm{~m}, 1 \mathrm{H})$, 12.03 (bs, 1H). LC-MS (ESI) $m / z\left[\mathrm{M}^{+}+\mathrm{H}\right]=442.1 . \mathrm{C}_{26} \mathrm{H}_{20} \mathrm{~N}_{2} \mathrm{O}_{5}$ ([M+H]) 440.9, found: 441.1; Elemental analysis calcd. (found) \%: C 70.18 (70.61); H 4.03 (4.35); N 6.83 (6.36).

1,2-Dihydro-4-((4-hydroxy-2-oxo-2H-chromen-3-yl)(3-hydroxyphenyl)methyl)-5-methyl-2-phenyl-1 $\boldsymbol{H}$-pyrazol3(2H)-3-one (5h): Yield: $82 \%$, white solid, m.p.: 260-264 ${ }^{\circ} \mathrm{C}$, IR $\left(\mathrm{KBr}, \mathrm{v}_{\max }, \mathrm{cm}^{-1}\right)$ : 3581, 1626, 1637, 1566, 1419, 1187, 1040, 754. ${ }^{1} \mathrm{H}$ NMR (400 MHz, DMSO) $\delta$ ppm: 2.36 (s, 3H), 5.70 (s, 1H), 6.80-6.86 (m, 2H), 7.09-7.19 (m, 5H), 7.31-7.42 (m, 3H), 7.57-7.60 (d, $J=8.1 \mathrm{~Hz}, 2 \mathrm{H}), 7.76-7.79$ (m, 1H), 12.03 (bs, 1H). LC-MS (ESI) $m / z\left[\mathrm{M}^{+}+\mathrm{H}\right]=442.1 . \mathrm{C}_{26} \mathrm{H}_{20} \mathrm{~N}_{2} \mathrm{O}_{5}([\mathrm{M}+\mathrm{H}])$ 440.9, found: 491.1; Elemental analysis calcd. (found) \%: C 71.18 (70.61); H 4.63 (4.35); N 6.03 (6.36).

1,2-Dihydro-4-((4-hydroxy-2-oxo-2H-chromen-3-yl)(4-methoxyphenyl)methyl)-5-methyl- 2phenyl- $\boldsymbol{H}$-pyrazol3(2H)-3-one (5i): Yield: $85 \%$, yellow crystalline solid, m.p. 199-202 ${ }^{\circ} \mathrm{C}$, IR (KBr, $\left.v_{\max }, \mathrm{cm}^{-1}\right)$ : 2989, 1693, 1614, 1566, 1512, 1450, 1151, 1021, 756. ${ }^{1} \mathrm{H}$ NMR (400 MHz, DMSO) $\delta$ : $2.38(\mathrm{~s}, 3 \mathrm{H}), 3.99(\mathrm{~s}, 3 \mathrm{H}), 4.22(\mathrm{~s}, 1 \mathrm{H}), 6.57-6.59(\mathrm{~m}, 3 \mathrm{H})$, 7.14-7.19 (m, 5H), 7.31-7.43(m, 3H), 7.51-7.55 (d, $J=8.1 \mathrm{~Hz}$, $2 \mathrm{H}), 7.78-7.80$ (d, $J=7.8 \mathrm{~Hz}, 1 \mathrm{H}) \mathrm{ppm}, \mathrm{LC}-\mathrm{MS}$ (ESI) $\mathrm{m} / \mathrm{z}$ $[\mathrm{M}+\mathrm{H}]=419.1 ; \mathrm{C}_{27} \mathrm{H}_{22} \mathrm{~N}_{2} \mathrm{O}_{4} \quad([\mathrm{M}+\mathrm{H}])$ 454.1, found: 454.1 . Elemental analysis calcd. (found) \%: C 71.85 (71.38); H 4.18 (4.91); N 6.06 (6.18).

1,2-Dihydro-4-((4-hydroxy-2-oxo-2H-chromen-3-yl)(2,3-dimethoxyphenyl)methyl)-5-methyl-2-phenylpyrazol3-one (5j): Yield: $89 \%$, pale yellow crystalline solid, m.p.: 223-225 ${ }^{\circ} \mathrm{C}$, IR (KBr, $\left.v_{\max }, \mathrm{cm}^{-1}\right)$ : 2934, 1666, 1613, 1477, 1277, 1181, 1071, 753. ${ }^{1} \mathrm{H}$ NMR (400 MHz, DMSO) $\delta$ ppm: 2.35 (s, 3H), 3.53 (s, 3H), 3.69 (s, 3H), 5.82 (s, 1H), 6.737.02 (m, 3H), 7.14-7.38 (m, 6H), 7.61 (bs, 2H), 7.74-7.76 (d, J $=7.8 \mathrm{~Hz}, 2 \mathrm{H})$. LC-MS (ESI) $m / z\left[\mathrm{M}^{+}+\mathrm{H}\right]=484.1 ; \mathrm{C}_{28} \mathrm{H}_{24} \mathrm{~N}_{2} \mathrm{O}_{6}$ $([\mathrm{M}+\mathrm{H}]=484.1$, found: 484.3. Elemental analysis calcd. (found) \%: C 69.41 (69.44); H 4.99 (5.03); N 5.78 (5.80). 


\section{RESULTS AND DISCUSSION}

As a part of the current ongoing studies devoted to the development of practical, safe and environmentally friendly procedures for synthesizing several important biologically active organic compounds, a simple and highly efficient route was used for the synthesis of benzylpyrazolyl coumarins as shown in Scheme-I.

First, to recognize the optimization of the reaction conditions, phenyl hydrazine $(1.0 \mathrm{mmol})$, ethyl acetoacetate $(1.0$ $\mathrm{mmol}), 3$-nitrobenzaldehyde $(1.0 \mathrm{mmol})$ and 4-hydroxycoumarin $(1.0 \mathrm{mmol})$ were, carrying out the reaction without using any catalyst. Table- 1 shows that the reaction completed in a long period of time (300 $\mathrm{min}$ ) and it was very sluggish and the yield of product was very low (10\%) (Table-1, entry 1). Secondly, for examining and finding out the ideal amounts of the catalysts $\mathrm{NbCl}_{5}$ and $\mathrm{AgClO}_{4}$, added to the reaction, the reaction was carried out several times in each which specific amounts of $\mathrm{NbCl}_{5}$ and $\mathrm{AgClO}_{4}$ were added. Table- 1 shows that the best amounts were $1 \mathrm{~mol} \%$ of $\mathrm{NbCl}_{5}$ and $3 \mathrm{~mol} \%$ of $\mathrm{AgClO}_{4}$; the reaction completed in the shortest period of time $(30 \mathrm{~min})$ and the yield of the product was excellent $(90 \%)$ (entry 6).
TABLE-1

OPTIMIZATION OF THE AMOUNT OF $\mathrm{NbCl}_{5}$ AND ADDITIVE FOR BENZYLPYRAZOLYL COUMARIN DERIVATIVES

\begin{tabular}{ccccc}
\hline Entry & $\begin{array}{c}\mathrm{NbCl}_{5} \\
(\mathrm{~mol} \%)\end{array}$ & $\begin{array}{c}\text { Additive } \\
(\mathrm{mol} \%)\end{array}$ & $\begin{array}{c}\text { Time } \\
(\mathrm{min})\end{array}$ & Yield $(\%)$ \\
\hline 1 & No catalyst & No catalyst & 300 & 10 \\
2 & 1 & - & 300 & 40 \\
3 & - & 1 & 300 & 25 \\
4 & 5 & - & 100 & 70 \\
5 & - & 5 & 150 & 58 \\
6 & 1 & 3 & 30 & 90 \\
7 & 3 & 3 & 30 & 88 \\
8 & 5 & 5 & 30 & 88 \\
\hline
\end{tabular}

Moreover, the efficiency of current method was evaluated by utilizing an extensive variety of aromatic aldehydes whenever electron withdrawing and electron donating groups for synthesis of the corresponding benzyl pyrazolyl coumarin derivatives. The reaction successes efficiently provided excellent yields and tolerated many electron-withdrawing and electrondonating substituted benzaldehydes (Table-2, entries 1-10).

Finally, we examined the advantage of our catalyst in synthesis of benzyl pyrazolyl coumarin derivatives used in

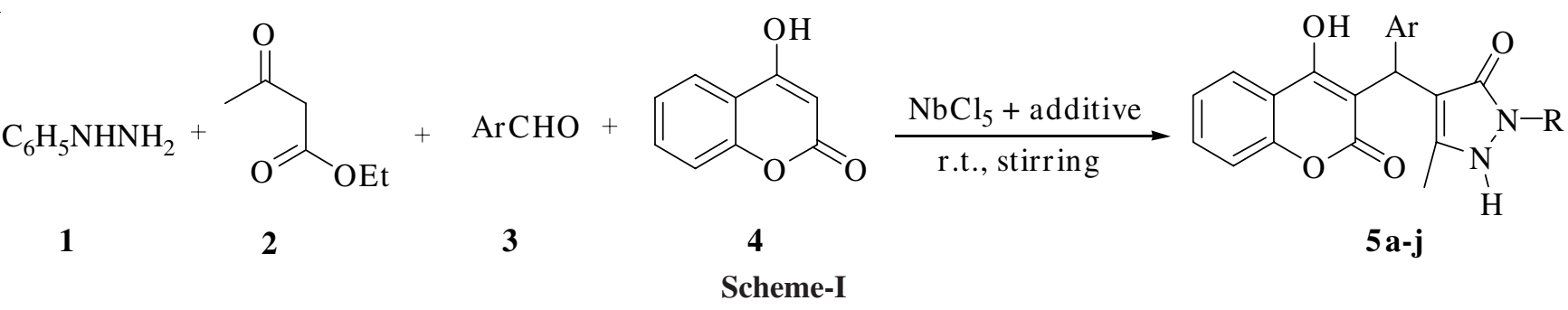

Entry Hydrazines




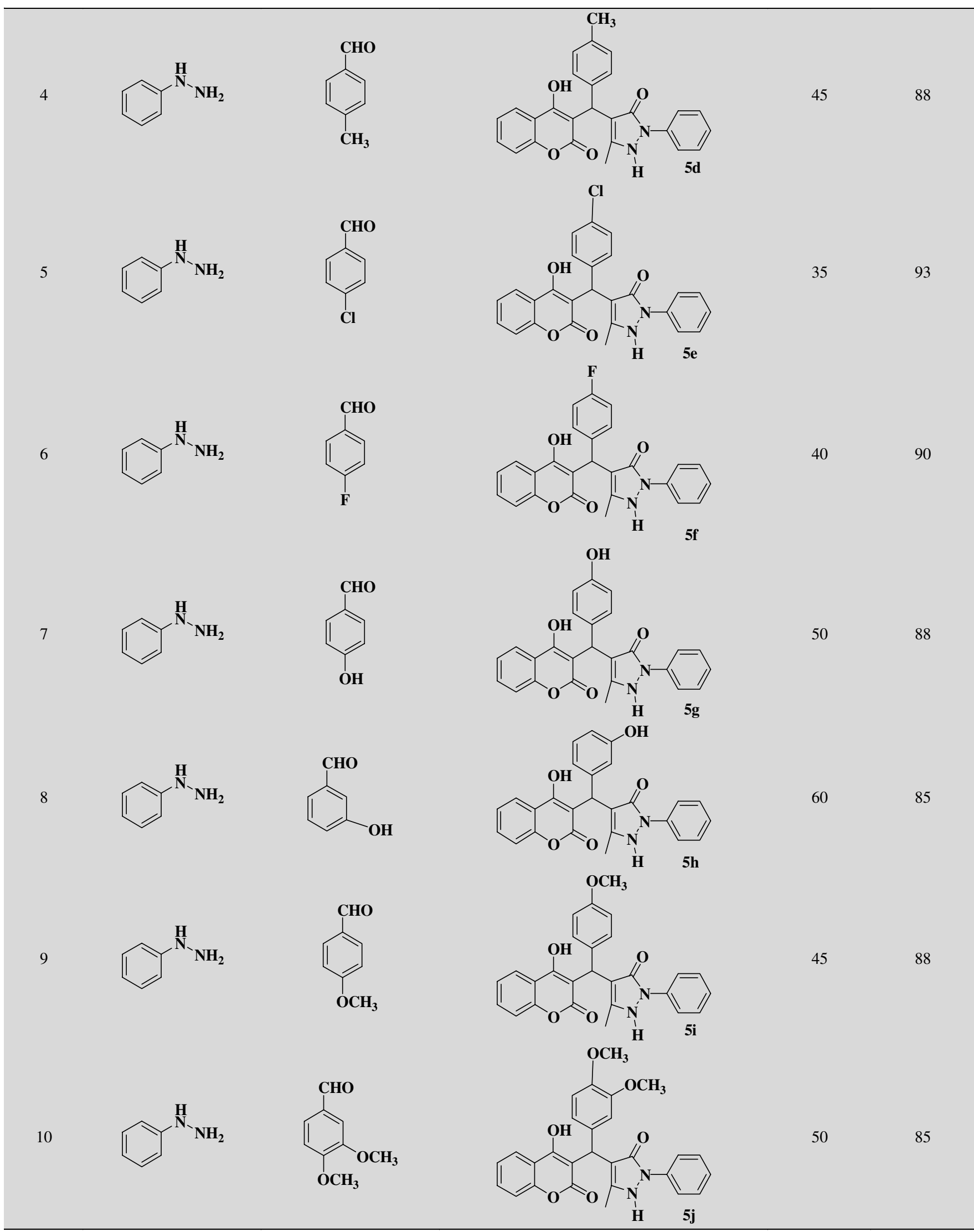

the present work to comparison with other results reported in the literature review to show the advantages of our catalyst under room temperature condition. the reactants; phenyl hydrazine $(1.0 \mathrm{mmol})$, ethyl acetoacetate $(1.0 \mathrm{mmol})$, benzaldehyde
(1.0 mmol) and 4-hydroxycoumarin (1.0 mmol) and $1 \mathrm{~mol} \%$ of $\mathrm{NbCl}_{5}$ and $3 \mathrm{~mol} \%$ of $\mathrm{AgClO}_{4}$ as catalysts using solvent free condition comparing with different catalysts as $\mathrm{Alg}(\mathrm{ACOH})$ [28] and $\mathrm{Ca}(\mathrm{OTf})_{2}[29]$ under reflux conditions. After compa- 
ring the combined acid catalyst under various conditions (room temp., reflux) with these catalysts, it was found that the combined acid catalyst under neat condition was the best catalyst for synthesis 1,2-dihydro-4-((4-hydroxy-2-oxo-2H-chromen-3-yl) (phenyl) methyl)-5-methyl-2-phenyl $1 H$-pyrazol-3(2H)-one with respect to the reaction time and the product yield.

\section{CONFLICT OF INTEREST}

The authors declare that there is no conflict of interests regarding the publication of this article.

\section{REFERENCES}

1. A. Domling, Chem. Rev., 106, 17 (2006); https://doi.org/10.1021/cr0505728.

2. J. Zhu and H. Bienayme, Multicomponent Reaction, Wiley-VCH: Weinheim (2005).

3. D.J. Ramón and M. Yus, Angew. Chem. Int. Ed., 44, 1602 (2005); https://doi.org/10.1002/anie.200460548.

4. A. Chanda and V.V. Fokin, Chem. Rev., 109, 725 (2009); https://doi.org/10.1021/cr800448q.

5. M.C. Pirrung and K.D. Sarma, J. Am. Chem. Soc., 126, 444 (2004); https://doi.org/10.1021/ja038583a.

6. C.-J. Li, Chem. Rev., 93, 2023 (1993); https://doi.org/10.1021/cr00022a004.

7. C.-J. Li, Chem. Rev., 105, 3095 (2005); https://doi.org/10.1021/cr030009u.

8. K. Tanaka and F. Toda, Chem. Rev., 100, 1025 (2000); https://doi.org/10.1021/cr940089p.

9. S. Hesse and G. Kirsch, Tetrahedron Lett., 43, 1213 (2002); https://doi.org/10.1016/S0040-4039(01)02373-5.

10. B.H. Lee, M.F. Clothier, F.E. Dutton, G.A. Conder and S.S. Johnson, Bioorg. Med. Chem. Lett., 8, 3317 (1998); https://doi.org/10.1016/S0960-894X(98)00588-5.

11. J.-C. Jung, Y.-J. Jung and O.-S. Park, Synth. Commun., 31, 1195 (2001); https://doi.org/10.1081/SCC-100104003.

12. G. Melagraki, A. Afantitis, O. Igglessi-Markopoulou, A. Detsi, M. Koufaki, C. Kontogiorgis and D.J. Hadjipavlou-Litina, Eur. J. Med. Chem., 44, 3020 (2009); https://doi.org/10.1016/j.ejmech.2008.12.027.

13. J.-C. Jung, J.-H. Lee, S. Oh, J.-G. Lee and O.-S. Park, Bioorg. Med. Chem. Lett., 14, 5527 (2004); https://doi.org/10.1016/j.bmcl.2004.09.009.
14. M. Himly, B. Jahn-Schmid, K. Pittertschatscher, B. Bohle, K. Grubmayr, F. Ferreira, H. Ebner and C.J. Ebner, Allergy Clin. Immunol., 111, 882 (2003); https://doi.org/10.1067/mai.2003.163.

15. T. Watanabe, S. Yuki, M. Egawa and H. Nishi, J. Pharmacol. Exp. Ther., 268, 1597 (1994).

16. H. Kawai, H. Nakai, M. Suga, S. Yuki, T. Watanabe and K.I. Saito, $J$. Pharmacol. Exp. Ther, 281, 921 (1997).

17. T.W. Wu, L.H. Zeng, J. Wu and K.P. Fung, Life Sci., 71, 2249 (2002); https://doi.org/10.1016/S0024-3205(02)01965-3.

18. M.A. Al-Haiza, S.A. El-Assiery and G.H. Sayed, Acta Pharm., 51, 251 (2001).

19. D. Castagnolo, F. Manetti, M. Radi, B. Bechi, M. Pagano, A. De Logu, R. Meleddu, M. Saddi and M. Botta, Bioorg. Med. Chem., 17, 5716 (2009); https://doi.org/10.1016/j.bmc.2009.05.058.

20. M. Radi, V. Bernardo, B. Bechi, D. Castagnolo, M. Pagano and M. Botta, Tetrahedron Lett., 50, 6572 (2009); https://doi.org/10.1016/j.tetlet.2009.09.047.

21. F. Moreau, N. Desroy, J.M. Genevard, V. Vongsouthi, V. Gerusz, G. Le Fralliec, C. Oliveira, S. Floquet, A. Denis, S. Escaich, M. Busemann, K. Wolf and A. Aschenbrenner, Bioorg. Med. Chem. Lett., 18, 4022 (2008); https://doi.org/10.1016/j.bmcl.2008.05.117.

22. F.A. Pasha, M. Muddassar, M.M. Neaz and S.J. Cho, J. Mol. Graph. Model., 28, 54 (2009); https://doi.org/10.1016/j.jmgm.2009.04.006.

23. C.E. Rosiere and M.I. Grossman, Science, 113, 651 (1951); https://doi.org/10.1126/science.113.2945.651.

24. D.M. Bailey, P.E. Hansen, A.G. Hlavac, E.R. Baizman, J. Pearl, A.F. Defelice and M.E. Feigenson, J. Med. Chem., 28, 256 (1985); https://doi.org/10.1021/jm00380a020.

25. P.M.S. Chauhan, S. Singh and R.K. Chatterjee, Indian J. Chem., 32B, 858 (1993).

26. P. Gunasekaran, S. Perumal, P. Yogeeswari and D. Sriram, Eur. J. Med. Chem., 46, 4530 (2011); https://doi.org/10.1016/j.ejmech.2011.07.029.

27. P.P. Ghosh, G. Pal, S. Paul and A.R. Das, Green Chem., 14, 2691 (2012); https://doi.org/10.1039/c2gc36021g.

28. S. Yaragorla, A. Pareek and R. Dada, Tetrahedron Lett., 56, 4770 (2015); https://doi.org/10.1016/j.tetlet.2015.06.049.

29. R.K. Pardeshi, S.A. Jadhav, M.G. Shioorkar, A.P. Sarkate and D.B. Shinde, Chem. Mater. Res., 7, 105 (2015). 